Published in final edited form as:

Lovis, V., Stefaniu, C., \& Brezesinski, G. (2019). Modification of Gibbs monolayers by chromium (III) compounds. Colloids and Surfaces A: Physicochemical and Engineering Aspects, 576, 29-35. doi:10.1016/j.colsurfa.2019.05.021.

\title{
Modification of Gibbs Monolayers by Chromium (III) Compounds
}

Victoria Lovisa, Cristina Stefaniu, Gerald Brezesinski
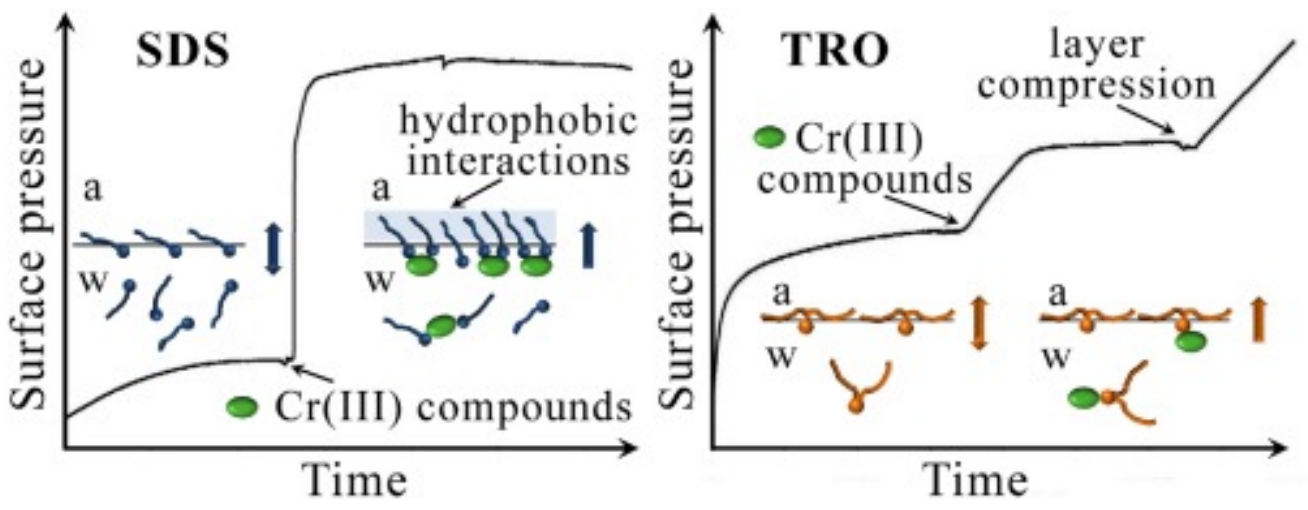


\title{
Modification of Gibbs Monolayers by Chromium (III) Compounds
}

\author{
Victoria Lovis ${ }^{\mathrm{a}, 1, *}$, Cristina Stefaniu ${ }^{\mathrm{a}}$, Gerald Brezesinski ${ }^{\mathrm{a}}$ \\ ${ }^{a}$ Max Plank Institute of Colloids and Interfaces, Science Park Potsdam-Golm, Am Mühlenberg 1, \\ 14476 Potsdam, Germany
}

\begin{abstract}
The present paper reveals the effects produced by a chromium (III) sulfate colloidal solution on Gibbs monolayers of (i) sodium dodecyl sulfate (SDS) and (ii) turkey red oil (TRO) formed at the air/water interface. Their intermolecular interactions and the modifications of the interphase properties play a significant role in the stabilization of concentrated reversed emulsions which have high relevance for the leather tanning technology. The changes produced in the amphiphilic monolayers were monitored employing highly sensitive surface characterization techniques such as Brewster angle microscopy (BAM), infrared reflection-absorption spectroscopy (IRRAS) and a Langmuir balance. The experimental data leads to the conclusion that amphiphile - chromium (III) complexes are formed. These complexes are characterized by an increased surface activity. In the case of the SDS containing system, the packing density increases and the hydrophobic interactions promote the formation of a stiff and brittle interface layer. Yet, only a loose packing is observed in the TRO monolayers, probably due to steric hindrance of
\end{abstract}

\footnotetext{
${ }^{*}$ Author to whom correspondence should be addressed.

Email address: victoria_lovis@outlook.com (Victoria Lovis)

${ }^{1}$ Max Plank Institute of Colloids and Interfaces, Science Park Potsdam-Golm, Am Mühlenberg 1, 14476 Potsdam, Germany
} 
the bulky hydrophobic chains.

Keywords: sodium dodecyl sulfate (SDS), chromium (III), Gibbs monolayer, turkey red oil (TRO), adsorption isotherm, BAM, IRRAS

\section{Introduction}

The present study focuses on defining the interactions between chromium (III) compounds (comprising the products of hydrolysis of chromium (III) sulfate: chromium (III) hydroxide and dihydroxide cations) and sodium dodecyl sulfate (SDS) or turkey red oil (TRO) amphiphiles adsorbed from aqueous solutions at the air/water interface.

Such interactions are crucial for the stabilization mechanism of emulsions containing chromium (III) sulfate colloidal solution as a dispersed phase and TRO as an external phase $[1,2]$. A technical analog of these emulsions is currently under development for utilization in leather tanning processes. In order to further optimize the technology of these emulsions, a deeper understanding of the stabilization mechanisms is needed. SDS, used as emulsifier in this system, is not capable to stabilize a reversed emulsion. Therefore, another component should play the role of the emulsion stabilizer.

To study the possible formation of the reversed emulsion stabilizer as a product of interaction between the emulsion components, the air/water interface has been used as a convenient model interface and the following experiments have been conducted. First of all, the effect of chromium (III) compounds on the adsorption of (i) SDS and (ii) TRO amphiphiles at the air/water interface has been quantified by recording the variation of the surface pressure with time. Then, Brewster angle microscopy (BAM) has been employed to monitor the morphology of the 
interfaces for both systems under study. Finally, infrared reflection-absorption spectroscopy (IRRAS) has been used to prove the influence of the interactions between the different components of the systems on layer thickness/packing efficiency.

\section{Experimental}

\subsection{Materials}

Chromium (III) sulfate colloidal solution is prepared by reducing potassium dichromate (chromium (VI)) (CAS: 7778-50-9, $\geq 99.5 \%$, ReagentPlus ${ }^{\circledR}$ grade, Sigma-Aldrich) with formaldehyde solution (CAS: 50-00-0, $37.4 \%$, grade for molecular biology, Sigma) in acidic media. All chemicals are used as received. Deionized water with conductivity less than $0.055 \mu \mathrm{S} / \mathrm{cm}$ is used as solvent. The acidic conditions ( $\mathrm{pH} 4)$ are realized by adding sulfuric acid (CAS: 7664-93-9, 95-98 \%, grade puriss p.a., Sigma-Aldrich). The ratio of the chemicals for the reaction is as follows: for $1.0 \mathrm{~g}$ of potassium dichromate, $1.0 \mathrm{ml}$ of distilled water, $0.38 \mathrm{ml}$ of formaldehyde solution and $0.52 \mathrm{ml}$ of sulfuric acid are supplied [3]. A chromium (III) concentrate is obtained from the reaction and diluted to $2.93 \mathrm{M}$ of chromium (III). Two kinds of titrimetric analyses are carried out in order to prove the negligibility of residual chromium (VI) and to verify the chromium (III) concentration in the prepared solution [3]. The colloidal solution is stored for at least two weeks prior to use. It is known that characteristics of the colloidal particles (such as colloidal particle size, PDI and zeta-potential) in chromium (III) sulfate colloidal solutions depend significantly on the preparation conditions (nature of reducing agent, $\mathrm{pH}$, etc.) as well as on the final concentration and dwell time. For instance, the colloidal particles in the systems obtained by dissolving chromium (III) hydroxide sulfate are characterized by a nearly even particle size distribution 
in the range from 21 to $49 \mathrm{~nm}$ after 40 days period of dwell for a chromium (III) concentration of $0.39 \mathrm{M}$ [4]. The colloidal solution used in this study is known to be stable towards sedimentation over several years.

Turkey red oil (sodium salt of sulfated castor-oil; CAS: 8002-33-3, grade for microscopy, Sigma-Aldrich) is used as received. It has appearance of a tawny, transparent liquid with high viscosity. According to the specification it contains 16-20\% water (Karl Fisher); the $\mathrm{pH}$ of a $10 \%$ solution is about 7-8. Turkey red oil can be obtained by the action of concentrated sulfuric acid on castor oil and dissolving the products in soda solution. It is a very complicated mixture of derivatives of recinoleic acid $\left(\mathrm{C}_{17} \mathrm{H}_{32}(\mathrm{OH}) \mathrm{COOH}\right)$. It contains (in the form of salts): free ricinoleic acid, its sulfuric acid ester (recinoleic acid sulfate) and the sulfuric acid ester of castor oil; perhaps also a mixed diricinoleic-acid-sulphuric glyceride, sulphuric ester of dioxystearic acid, ricinoleic acid anhydride, polyricinoleic acid, and other minor compounds [5].

Sodium dodecyl sulfate (CAS: 151-21-3, grade ultra for molecular biology, Fluka) is used as received.

\subsection{Methods}

\subsubsection{Surface pressure vs. time isotherms}

Adsorption of SDS or TRO amphiphiles at the air/water interface from their aqueous solutions as well as the effect of chromium (III) compounds added subsequently to the subphase of each of the systems are quantified via surface pressure vs. time isotherms. The isotherms are recorded according to the Wilhelmy method $[6,7,8]$ which relates the accumulation of the surface active molecules at the interface to the alteration of the surface tension with time.

The method uses a Langmuir balance consisting of a force measuring system 
and a homemade trough covered with a polytetrafluorethylene (PTFE) foil and equipped with mobile PTFE barriers. The force measuring system includes electronic weighting device (Standard-Filmwaage, Germany) and a glass plate. The instrument is mounted onto an anti-vibrating table (Halcyonics, Germany). The accuracy of the surface pressure measurement is $\pm 0.02 \mathrm{mN} / \mathrm{m}$. The instrument operation and data acquisition are performed via computer software FW 2, Version 1.1.0. The temperature of the subphase in the trough is maintained by means of a circulation thermostat (RC 6 CP Lauda, Germany) with the precision of the heat conductor (water) temperature $\pm 1.0^{\circ} \mathrm{C}$.

To acquire the experimental data, the trough is filled with either $1.0 \mathrm{mM}$ aqueous solution of SDS or 158 fold diluted with water TRO (which corresponds to approximately $0.005 \mathrm{mM}$ assuming all TRO (anhydrous) consists of sodium salt of sulfated castor oil). These concentrations have been chosen based on the results of preliminary studies (not presented here) since they ensure a measurable effect of chromium (III) compound on the systems and are experimentally admissible. These concentrations result in equilibrium surface pressure $11.2 \mathrm{mN} / \mathrm{m}$ and 16.6 $\mathrm{mN} / \mathrm{m}$ for SDS and TRO monolayers, correspondingly. The temperature of the subphase is maintained at $(20 \pm 1){ }^{\circ} \mathrm{C}$ by means of the circulation water bath. The water phase level is adjusted in a way that the calibrated Wilhelmy glass plate is immersed into the liquid and the measured surface pressure is adjusted to $(0.00 \pm 0.02) \mathrm{mN} / \mathrm{m}$. The surface pressure is measured every $0.2 \mathrm{~min}$. After reaching equilibrium of the SDS or TRO adsorption (the Gibbs monolayer at the air/water interface is formed indicated by the plateau in the surface pressure vs. time diagram), the chromium (III) sulfate colloidal solution is added gently to the subphase (as shown in fig. 1) giving a final concentration of $9 \mathrm{mM}$ of chromium 
(III) in the subphase. The experimental data are continuously acquired.

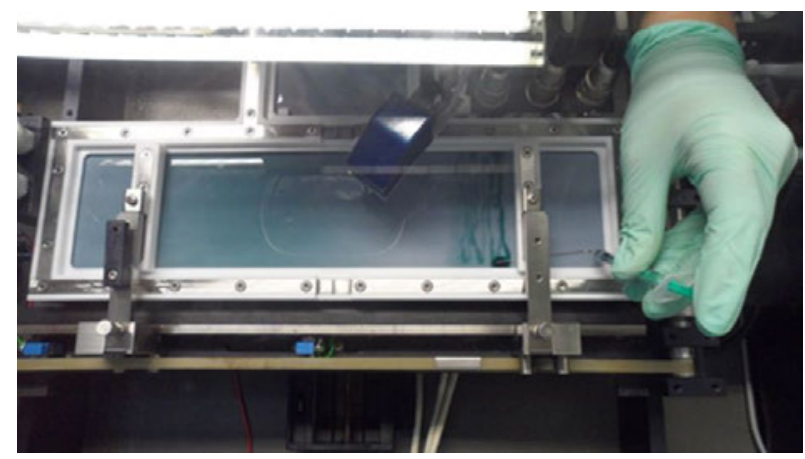

Figure 1: Adding chromium (III) sulfate colloidal solution to the subphase of the system under study.

\subsection{2. $B A M$}

The morphology of the interface is visualized by Brewster angle microscopy (BAM) $[9,10,11]$. The method is based on differences between the refractive index of the liquid media (subphase) and the refractive index of the interfacial medium (monolayer at the interface). As a result, the p-polarized laser beam, which incidents the subphase at the Brewster angle, is partly reflected from the monolayer covered surface. The reflected light can be detected and one obtains a micrograph of the bright domains on the dark background. Thus, any film having a refractive index different from that of a subphase can be observed.

In this study, Brewster angle microscope BAM1+ (NFT, Göttingen, Germany) coupled with the Langmuir balance described above is used. The incident laser beam $(\lambda=632.8 \mathrm{~nm}, 10 \mathrm{~mW})$ hits the surface after passing through a polarizer (p-polarized light is used) at the Brewster angle. The micrographs are captured with a lateral resolution of $4 \mu \mathrm{m}$.

A monolayer of SDS or TRO is formed according to the procedure described 
in 2.2.1. For each system, the BAM micrographs are acquired at the Brewster angle of water as soon as the adsorption equilibrium is reached. Subsequently, the chromium (III) sulfate colloidal solution is added to the subphase giving a final concentration of $9 \mathrm{mM}$ of chromium (III). BAM micrographs are acquired again. For the system containing TRO, additional micrographs are taken upon compression of the interfacial layer by means of displacement of the PTFE barriers.

\subsubsection{IRRAS}

Infrared reflection-absorption spectroscopy (IRRAS) [12, 13, 14, 15] is based on the specific absorption of electromagnetic waves leading to the vibration of atoms or groups of atoms. The decrease of the reflected intensity at the specific energy can be quantified.

The single-beam infrared reflectance spectra are recorded by means of a Vertex 70 FT-IR spectrometer (Bruker, Germany) equipped with a liquid nitrogen cooled MCT detector. The spectrometer is coupled with a Langmuir film balance identical to the one described above, which features, however, two compartments: one for the sample (monolayer) and one for a reference (bare air/water interface). The instrument setup is placed in a sealed container (an external air/water reflection unit XA-511, Bruker, Germany) to guarantee a constant vapor atmosphere.

The sample compartment of the trough is filled with an amphiphile solution (1.0 mM SDS or $0.005 \mathrm{mM}$ TRO). The reference compartment of the trough is filled correspondingly with distilled water. The temperature in the trough is maintained at $(20 \pm 1){ }^{\circ} \mathrm{C}$ by means of a circulation bath. After the monolayer at the surface of the amphiphile solutions has been formed, the chromium (III) sulfate colloidal solution is supplied as described above leading to a final concentration of $9 \mathrm{mM}$ of chromium (III) in the subphase. After certain periods of dwell, the 
spectra of the sample and the reference (used as background) are recorded at an incidence angle of $40^{\circ}$ using parallel (p) or vertically (s) polarized IR light. The spectra are recorded with $4 \mathrm{~cm}^{-1}$ resolution. A computer controlled "trough shuttle system" is used to switch between recording the sample and the reference spectra.

In order to eliminate the water signal, the reflection-absorption spectra are calculated as $-\log \left(\mathrm{R} / \mathrm{R}_{0}\right)$, where $\mathrm{R}_{0}$ and $\mathrm{R}$ are the reflectivities of the bare (reference) and the monolayer-covered (sample) surfaces, respectively. Thus, positive bands correspond to surface concentrations smaller than those in the reference, negative bands to higher ones. The resulting infrared reflection-absorption spectra are composed by co-adding 400 interferograms for s-polarized light and 800 interferograms for p-polarized light. The analysis of the resulting reflection-absorption spectra (position and intensity of vibrational bands) is performed by means of the Origin Pro software.

\section{Results and discussion}

In the following, the experimental results for the two systems under study, namely SDS and TRO monolayers formed at the corresponding amphiphile solution surface, are summarized and discussed. Particularly, modification of the interphase structure and properties upon adding chromium (III) compounds is elucidated.

\subsection{SDS monolayers}

The formation of SDS Gibbs monolayers (adsorption of SDS from its aqueous solution) is monitored by recording the surface pressure vs. time as described in subsection 2.2.1. After the equilibrium surface pressure $(11.2 \mathrm{mN} / \mathrm{m})$ has been reached, the chromium (III) sulfate colloidal solution is added to the subphase 
(subsection 2.2.1). The surface pressure starts immediately to increase, indicating an increased surface activity and tighter packing of SDS at the interface (fig. 2). The immediate increase of the surface pressure suggests that only highly mobile compounds of the colloidal solution (presumably chromium (III) hydroxide and dihydroxide cations) affect the interphase structure. After reaching the new plateau (about 37.0 to $39.6 \mathrm{mN} / \mathrm{m}$ ), the adsorption layer is compressed by means of the mobile barriers. Compression does not change the surface pressure significantly indicating that no denser packing of the amphiphile molecules can be reached. The unchanged lateral pressure could indicate that the adsorption layer is in thermodynamic equilibrium with the bulk phase (exchange of molecules between surface and bulk).

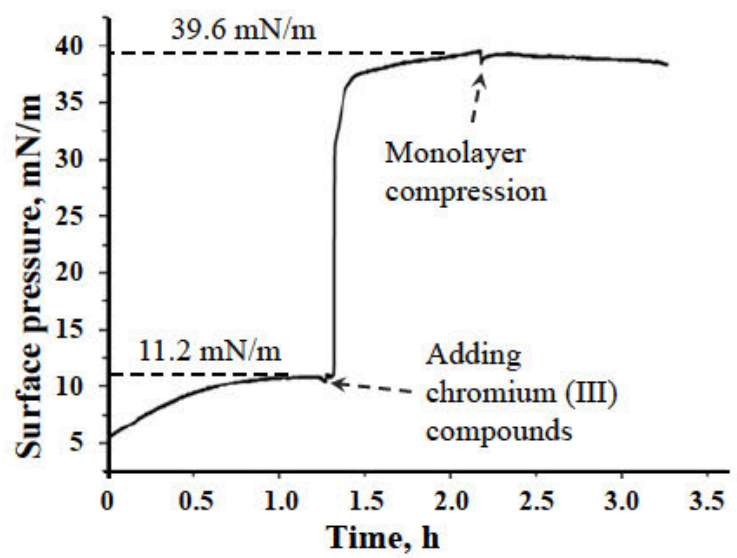

Figure 2: Surface pressure vs. time isotherm for the SDS monolayer: Gibbs monolayer formation, adding chromium (III) sulfate colloidal solution leading to an increased surface activity, and the layer compression.

The morphology of the interface is visualized by BAM according to the subsection 2.2.2. The micrograph of the surface at the equilibrium (plateau at 11.2 $\mathrm{mN} / \mathrm{m}$ (fig. 2), no chromium (III) compounds are supplied yet) presented in fig. 
3(a) reveals no inhomogeneities within the Gibbs layer (with the resolution limit of the BAM). However, after adding the chromium (III) sulfate colloidal solution to the subphase the first domains can be seen at the surface (fig. 3(b)). The domains grow and become brighter with time (fig. 3(c)). This indicates that the presence of chromium (III) compounds in the subphase leads to the formation of an inhomogeneous layer with densely packed parts (bright domains) which are thicker than the surrounding area. Moreover, upon compression of the interface layer by means of the mobile barriers the interphase breaks into pieces (not illustrated here) what suggests brittle mechanical properties. This contradicts our assumption of an apparent thermodynamical equilibrium based on no changes in lateral pressure during compression. Comparison isotherm and BAM results leads rather to a conception of SDS - chromium (III) complexes trapped at the interface.

Comparison of the infrared reflection-absorption spectra acquired before and after adding the chromium (III) compounds to the subphase (no compression) according to the procedure described in 2.2.3 reveals the following effects. First of all, the continuous significant increase of $-\log \left(R / R_{0}\right)$ in the range of the wave numbers corresponding to the hydroxyl absorption bands located at $1600-1700 \mathrm{~cm}^{-1}$ and $3000-3700 \mathrm{~cm}^{-1}$ (fig. 4) confirms an increase in the effective adsorption layer thickness (tighter packing/thicker layer). The growth of the negative bands corresponding to the symmetric and asymmetric stretching vibrations of the methylene groups $\left(2800-3000 \mathrm{~cm}^{-1}\right)$ as well as the symmetric and asymmetric vibrations of the sulfate group (900 - $1300 \mathrm{~cm}^{-1}$ ) indicates that in presence of chromium (III) compounds the amphiphile molecules are continuously adsorbed at the interface.

The shift of the methylene stretching bands to lower wave numbers (fig. 5) indicates a transition from a clearly liquid-expanded state with many gauche con- 

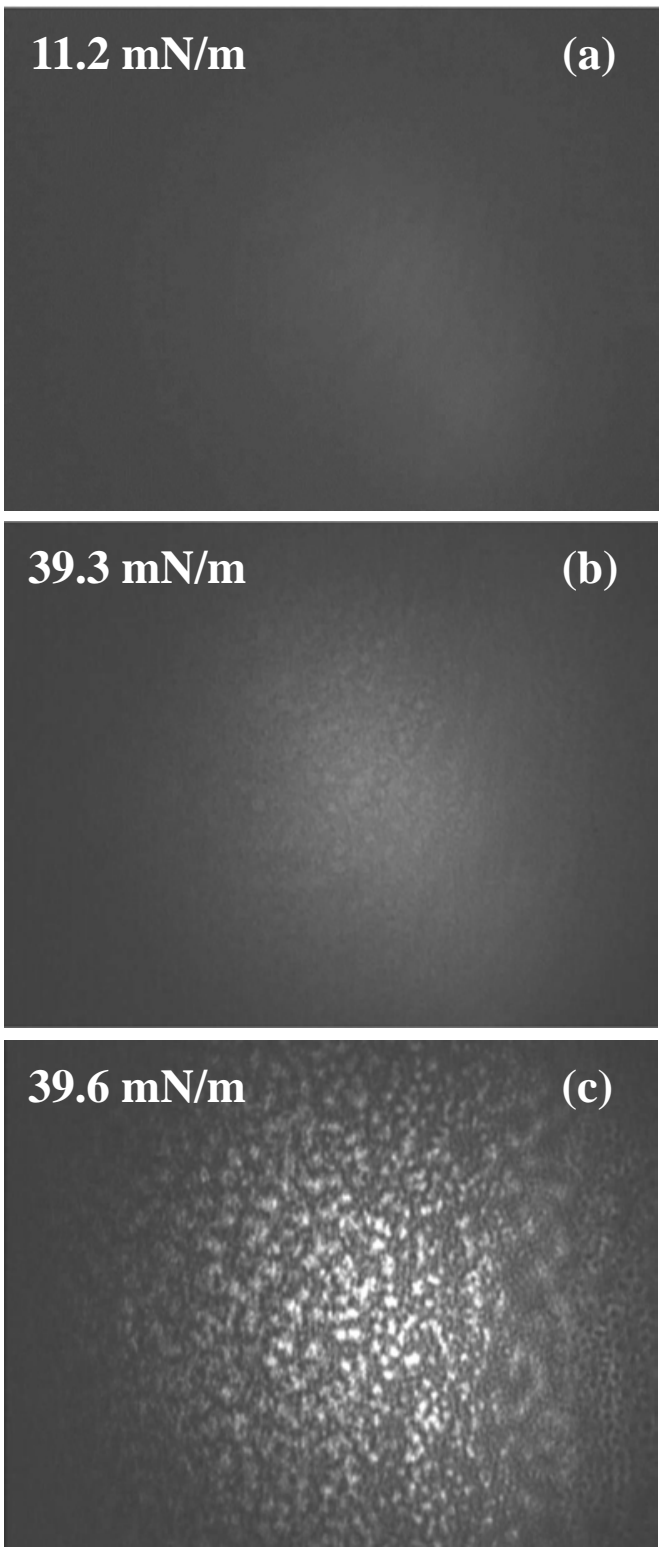

Figure 3: BAM micrographs of the SDS aqueous solution surface before (a) and after (b, c) adding chromium (III) compounds, no compression of the interface layer. The corresponding surface pressures are indicated. The size of each micrograph is about $470 \times 355 \mu \mathrm{m}^{2}$. 
formers $\left(\nu_{s} \sim 2855.2 \mathrm{~cm}^{-1}\right.$ and $\left.\nu_{a s} \sim 2925.0 \mathrm{~cm}^{-1}\right)$ to a more condensed state with some all-trans conformers $\left(\nu_{s} \sim 2852.9 \mathrm{~cm}^{-1}\right.$ and $\nu_{a s} \sim 2923.1 \mathrm{~cm}^{-1}$ after $150 \mathrm{~min}$ of dwell) $[16,17]$. It is interesting to note that the increase of the surface pressure is much faster than the decrease of the wave numbers of the methylene stretching bands. This shows that the molecules in the adsorption layer need a longer time to re-arrange into domains with a higher packing density (see fig. 3(c)).

Moreover, significant shifts of the peak positions for the symmetric (from $\sim$ 1071.9 to $\sim 1060.7 \mathrm{~cm}^{-1}$ ) and for the asymmetric (from $\sim 1217.2$ to $\sim 1197.0$ $\mathrm{cm}^{-1}$ ) vibration of the sulfate groups are observed after adding chromium (III) compounds to the subphase. The shift of the sulfate related bands to smaller wave numbers has been observed for SDS interacting with alumina (III) [18] or titanium (V) [19] oxides. The authors assign the effect to the adsorption of SDS to the metal oxides.

\subsection{TRO monolayers}

Investigation of the TRO monolayer formation by monitoring the surface pressure vs. time as described in subsection 2.2.1 shows a behavior similar to SDS. However, two significant differences can be seen by comparing figures 2 and 6 . First of all, the increase of the surface pressure after adding chromium (III) compounds to the subphase (fig. 6) is about three fold less than the increase in the case of the SDS containing system. The second important feature is that the surface pressure rises upon compression of the TRO adsorption layer. The latter means that the TRO monolayer is not densely packed after adding the chromium (III) compounds and the TRO molecules trapped at the surface are not in thermodynamic equilibrium with the bulk solution.

Observation of the interface morphology of the system containing TRO before 


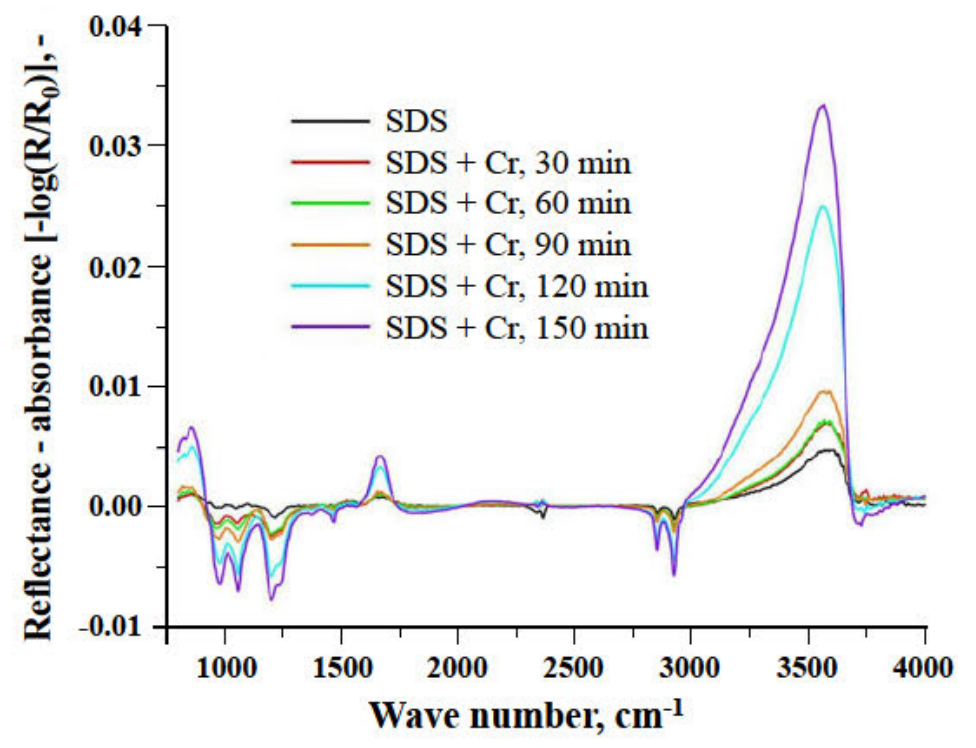

Figure 4: Infrared reflectance-absorbance spectra of the SDS covered surface before and after adding chromium (III) compounds to the subphase.

and after adding chromium (III) compounds by BAM is barely possible (fig. 7(a, b)). Only after decreasing the surface area by compression an inhomogeneous interface layer with thicker areas starts to form (fig. 7(c)). This behavior is clearly different compared to the behavior of the SDS containing system after adding the chromium (III) compounds.

The IRRAS measurements after adding chromium (III) compounds (no compression) (fig. 8) reveal only a slight increase of $-\log \left(R / R_{0}\right)$ for the hydroxyl band $\left(1600-1700 \mathrm{~cm}^{-1}\right.$ and $\left.3000-3700 \mathrm{~cm}^{-1}\right)$ compared to the system without chromium (III) compounds. In contrary to the SDS containing system, the intensity does not rise upon dwell. Also no important change is detected in the methylene stretching vibration range $\left(2800-3000 \mathrm{~cm}^{-1}\right)$. These small effects suggest no significant increase of the packing density and thickness of the adsorption layer 

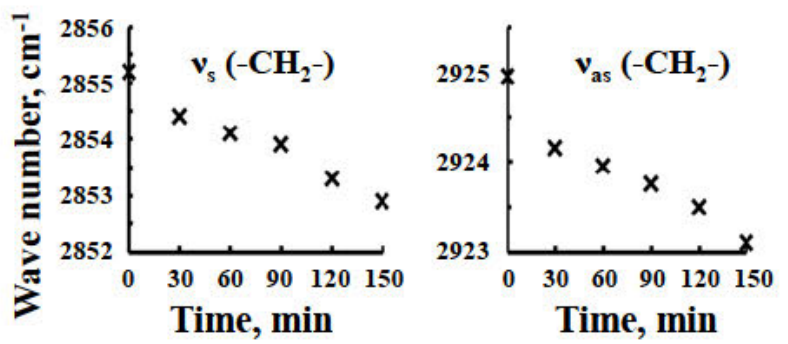

Figure 5: The position of the methylene symmetric (left) and asymmetric (right) stretching vibration bands vs. time.

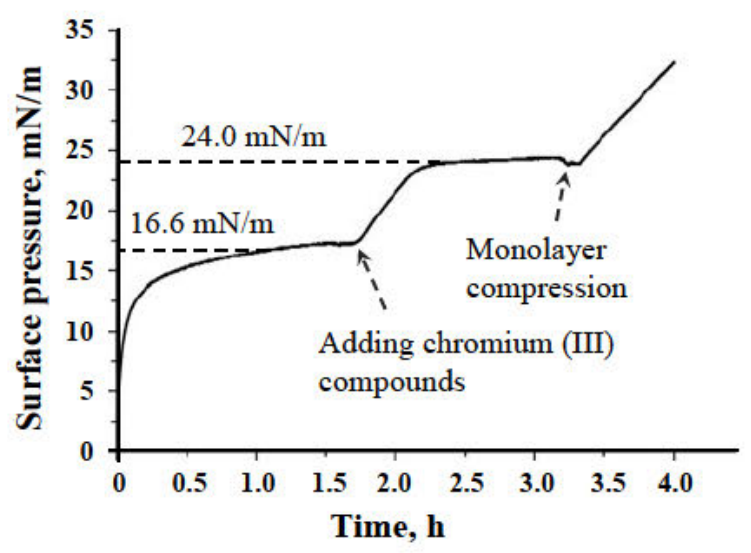

Figure 6: Surface pressure vs. time isotherm for the TRO monolayer: monolayer formation, adding chromium (III) sulfate colloidal solution, and layer compression.

after adding chromium (III) compounds to the subphase. A pronounced (negative) increase of $-\log \left(R / R_{0}\right)$ is observed, however, for the sulfate-related bands (at $850-1100 \mathrm{~cm}^{-1}$ ). This might indicate a change in the orientation of the transition dipole moment of the sulfate group due to certain interaction with the chromium (III) compounds $[16,17]$.

For SDS as well as for TRO the adsorption equilibria in absence of chromium (III) compounds are determined by the delicate balance between attraction (van 

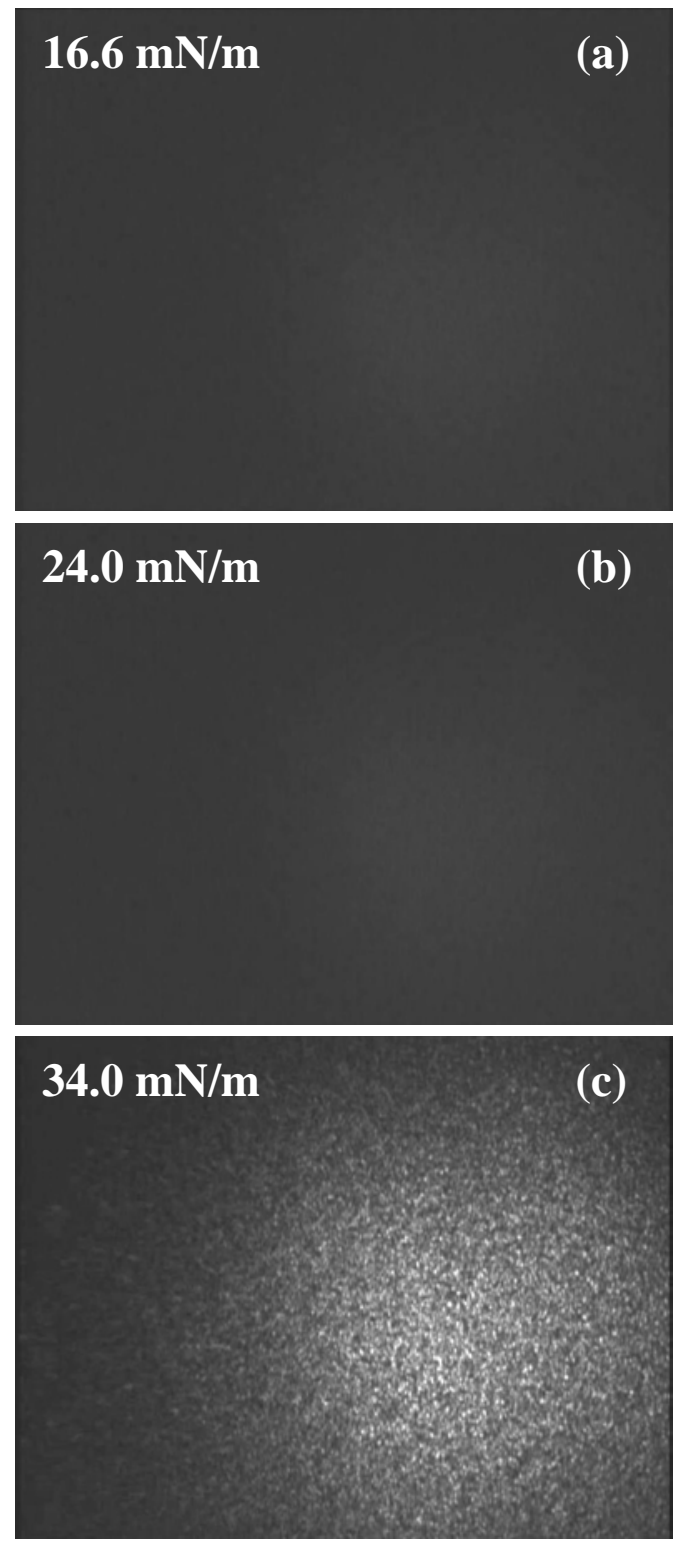

Figure 7: BAM micrographs of the TRO aqueous solution surface before (a) and after (b) adding chromium (III) compounds. Additionally, the morphology of the interface upon compression is presented (c). The corresponding surface pressures are indicated. The size of each micrograph is about $470 \times 355 \mu \mathrm{m}^{2}$. 


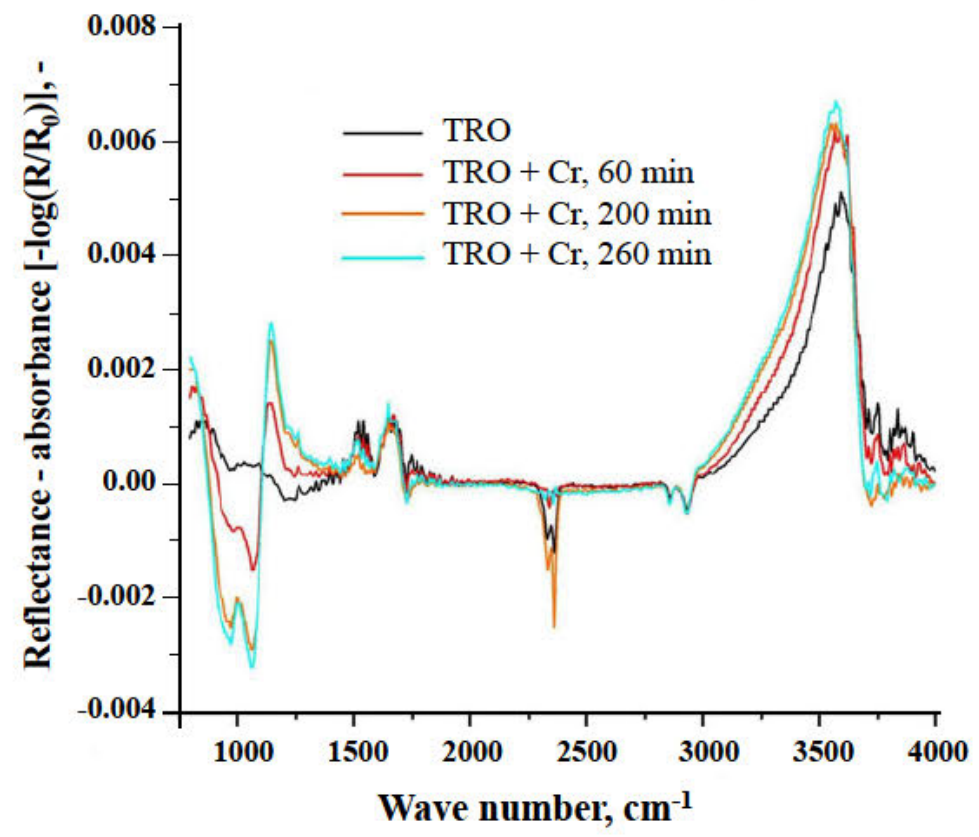

Figure 8: Infrared reflectance-absorbance spectra of the TRO covered surface before and after adding chromium (III) compounds to the subphase. 
der Waals forces between the hydrophobic chains, so called hydrophobic interaction) and repulsion (electrostatic forces between charged sulfate groups) at the interface [20], and the chemical potential of the amphiphile in the bulk. In the case of TRO monolayers, additional steric hindrance is expected to affect the equilibrium: even ricinoleic acid sulfate, being the smallest constituent surfactant species, carries its hydrophilic sulfate group at the center of the hydrocarbon chain, thus featuring two hydrophobic tails. The other sulfated TRO constituents are larger and thus even more bulky, leading to significant steric hindrance in an adsorption layer.

Adding the chromium (III) compounds induces a pronounced increase of the surface pressure (see figs. 2 and 6), pointing towards a significant increase of the surface activity of the amphiphilic species. Relying on IRRAS results (which exhibit changes in the sulfate related peaks position/intensity, see figs. 4 and 8) we conclude that due to interactions between the anionic sulfate groups and cationic chromium (III) species amphiphile - chromium (III) complexes are formed. These complexes are obviously characterized by an increased surface activity as compared with that of the free SDS and TRO amphiphile molecules.

Further analysis of the surface pressure vs. time isotherms (figs. 2 and 6) reveals two significant differences. First of all, the increase of the surface pressure after adding chromium (III) compounds to the subphase of the TRO containing system is about three times smaller than the increase in case of the SDS containing system. This might be explained by the significantly lower bulk concentration (and, therefore, lower chemical potential) of TRO surfactant molecules (about $0.005 \mathrm{mM}$ vs. $1.0 \mathrm{mM}$ for SDS). On the other hand, TRO amphiphile molecules are in general more surface active than SDS molecules. After the addition of 
chromium (III) compounds, the surface activity of the complexes formed increases in both cases. However, in the case of TRO the activity of the complex formed is only slightly higher than the activity of TRO amphiphiles. In the case of SDS, the activity of the complex formed is significantly higher than the activity of SDS and the adsorption increases drastically.

The second important feature is that in the presence of chromium (III) compounds the surface pressure rises upon compression of the TRO adsorption layer while it does not change upon compressing the SDS adsorption layer. The latter can be explained as follows. The adsorption of SDS is mainly limited by the electrostatic repulsion of the charged sulfate groups. In presence of chromium (III) compounds a SDS - chromium (III) complex is formed as discussed above. The negative charges are now screened more efficiently compared to the weaker interacting potassium or sodium ions. Therefore, a denser packing of the amphiphiles can be reached at the interface. Additionally, even more than one sulfate group can interact with a highly charged chromium (III) hydroxide cation. Such interactions facilitate further increase in the packing density and promote an increased order (condensation) of the hydrocarbon chains due to the hydrophobic interactions. As expected, this rather slow process of condensation (illustrated by the decrease of the wave numbers for methylene related stretching vibrational band positions, fig. 5) is accompanied by an increased surface concentration (evidenced for instance by increased intensities of the methylene related bands, fig. 4) at a virtually constant surface pressure on the second plateau (see fig. 2). Finally, the aligned dodecyl chains (tilted to the surface) locally increase the adsorption layer thickness which is directly observed in form of bright domains in the BAM micrographs (fig. 3(b,c)). The darker areas correspond to the interface covered with 
SDS still remaining in the loosely packed state. Indeed, compression first consolidates the interface layer: the amphiphile molecules are forced to approach each other closer and undergo further condensation. Such a strongly interlinked layer is expected to be stiff and brittle [21, 22]. Upon further compression the interphase breaks into pieces as observed by BAM. All of that happens at a constant surface pressure (see fig. 2).

On the other hand, adsorption of TRO amphiphile molecules is limited by both electrostatic repulsion of the charged sulfate groups and steric packing problems of the bulky hydrophobic parts. The charge screening by chromium (III) species can only decrease the electrostatic repulsion while the steric hindrance remains. Thus, the packing density is affected to a much lower extent compared to SDS. As a result, no condensation process driven by hydrophobic interactions of hydrocarbon chains can take place and no prolonged adsorption is observed after adding the chromium (III) compounds (see fig. 8). However, the amphiphile molecules are trapped at the surface and the steric hindrance in the disordered adsorbate structure can be overcome by mechanical force. In fact, mechanical compression enforces denser packing of the hydrophobic chains in a still liquid-like layer; the corresponding surface pressure rises (fig. 6).

\section{Conclusions}

In this study, the interactions of SDS and TRO with chromium (III) compounds are investigated at the model air/water interface by means of surface sensitive techniques such as the Langmuir balance, BAM and IRRAS.

From the observed effects it is derived that in both systems amphiphile chromium (III) complexes characterized by increased surface activity are formed, presumably due to interactions between the anionic sulfate groups and cationic 
chromium (III) species. In the case of the SDS containing system, the adsorption layer undergoes condensation, resulting in a strongly interlinked layer which is brittle upon compression. Yet, the monolayer of TRO remains loosely packed upon the interaction with the chromium (III) compounds, indicating that the condensation is limited by the steric hindrance of the bulky hydrophobic chains.

The knowledge obtained from this study can be used to better understand the mechanism of stabilization of the reversed emulsions consisting of chromium (III) sulfate colloidal solution, TRO, and SDS and which are of high relevance for the leather tanning technology.

\section{Acknowledgments}

The financial support provided by DAAD (Deutscher Akademischer Austauschdienst; postgraduate research stay) to V.L. is gratefully acknowledged. The authors thank sincerely Irina Berndt for assistance with experiment preparation. V.L. thanks the research group of Prof. Brezesinski for the warm work/research atmosphere and Dr. Florian Lovis for useful recommendations and constructive feedback on the manuscript.

\section{References}

[1] V. Krupennikova, V. Radnaeva, B. Tanganov, The study of the dependence of tanning disperse system's properties on it components' physical-chemical properties by applying the mathematical simulation methods, Colloids and Surfaces A: Physicochemical and Engineering Aspects 383 (2011) 86 - 89.

[2] V. Lovis, V. D. Radnaeva, G. Brezesinski, B. B. Tanganov, Reverse emulsions containing a chromium(III) sulfate colloidal solution: effects of quan- 
titative composition of the phases and some features of stabilization mechanisms, in preparation .

[3] A. A. Golovteeva, D. A. Kuzidi, L. B. Sankin, Laboratory Practicum on Chemistry and Technology of Leather and Fur [Russian], Moscow, 1987.

[4] S. V. Gorbatov, A. N. Mihailov, V. N. Kulagin, N. I. Lopatina, N. A. Nabokova, D. S. Lychnikov, Solutions of basic chromium (III) sults as colloidal systems [Russian], Leather and Footware Industry [Russian] (1985) $41-43$.

[5] A. Grün, M. Woldenberg, Investigation of the essential constituent of turkey red oil and its derivatives, Journal of the American Chemical Society 31 (1909) 490-506.

[6] G. Brezesinski, H. Möhwald, Langmuir monolayers to study interactions at model membrane surfaces, Advances in Colloid and Interface Science 100102 (2003) 563-584.

[7] H. Möhwald, G. Brezesinski, From Langmuir monolayers to multilayer films, Langmuir 32 (2016) 10445-10458.

[8] C. Stefaniu, G. Brezesinski, H. Möhwald, Langmuir monolayers as models to study processes at membrane surfaces, Advances in Colloid and Interface Science 208 (2014) 197-213.

[9] S. Hénon, J. Meunier, Microscope at the Brewster angle: direct observation of first-order phase transitions in monolayers, Review of Scientific Instruments 62 (1991) 936-939. 
[10] D. Hönig, D. Möbius, Direct visualization of monolayers at the air-water interface by Brewster angle microscopy, The Journal of Physical Chemistry 95 (1991) 4590-4592.

[11] D. Vollhardt, Morphology and phase behavior of monolayers, Advances in Colloid and Interface Science 64 (1996) 143-171.

[12] R. A. Dluhy, R. Mendelsohn, H. L. Casal, H. H. Mantsch, Interaction of dipalmitoylphosphatidylcholine and dimyristoylphosphatidylcholine- $\mathrm{d}_{54}$ mixtures with glycophorin. A Fourier transform infrared investigation, Biochemistry 22 (1983) $1170-1177$.

[13] R. Mendelsohn, J. W. Brauner, A. Gericke, External infrared reflection absorption spectrometry of monolayer films at the air-water interface, Annual Review of Physical Chemistry 46 (1995) 305-334.

[14] C. R. Flach, A. Gericke, R. Mendelsohn, Quantitative determination of molecular chain tilt angles in monolayer films at the air/water interface: infrared reflection/absorption spectroscopy of behenic acid methyl ester, The Journal of Physical Chemistry B 101 (1997) 58-65.

[15] A. H. Muenter, J. Hentschel, H. G. Börner, G. Brezesinski, Characterization of peptide-guided polymer assembly at the air/water interface, Langmuir 24 (2008) 3306-3316.

[16] R. Mendelsohn, C. R. Flach, Infrared reflection-absorption spectroscopy of lipids, peptides, and proteins in aqueous monolayers, Current Topics in Membranes 52 (2002) 57-88. 
[17] R. Mendelsohn, G. Mao, C. R. Flach, Infrared reflection-absorption spectroscopy: principles and applications to lipid - protein interaction in Langmuir films, Biochimica et Biophysica Acta 1798 (2010) 788-800.

[18] R. P. Sperline, Y. Song, H. Freiser, Fourier transform infrared attenuated total reflection spectroscopy linear dichroism study of sodium dodecyl sulfate adsorption at the $\mathrm{Al}_{2} \mathrm{O}_{3}$ /water interface using $\mathrm{Al}_{2} \mathrm{O}_{3}$-coated optics, Langmuir 8 (1992) 2183-2191.

[19] K. D. Dobson, A. D. Roddick-Lanzilotta, A. J. McQuillan, An in situ infrared spectroscopic investigation of adsorption of sodium dodecylsulfate and of cetyltrimethylammonium bromide surfactants to $\mathrm{TiO}_{2}, \mathrm{ZrO}_{2}, \mathrm{Al}_{2} \mathrm{O}_{3}$, and $\mathrm{Ta}_{2} \mathrm{O}_{5}$ particle films from aqueous solutions, Vibrational Spectroscopy 24 (2000) 287-295.

[20] S. I. Karakashev, A. V. Nguyen, J. D. Miller, Equilibrium adsorption of surfactants at the gas-liquid interface, Advances in Polymer Science 218 (2008) $25-55$.

[21] G. A. Wolstenholme, J. H. Schulman, Metal - monolayer interactions in aqueous systems. Part I. - The interaction of monolayers of long-chain polar compounds with metal ions in the underlying solution, Transactions of the Faraday Society 46 (1950) 475-487.

[22] P. Sundberg, M. Karppinen, Organic and inorganic - organic thin film structures by molecular layer deposition - A review, Beilstein Journal of Nanotechnology 5 (2014) 1104-1136. 